\title{
Erratum: Estimation of coherent error sources from stabilizer measurements [Phys. Rev. A 93, 042303 (2016)]
}

\author{
Davide Orsucci, Markus Tiersch, and Hans J. Briegel
}

(Received 24 April 2019; published 20 May 2019)

DOI: 10.1103/PhysRevA.99.059901

Equation (6) in the original article is as follows:

$$
\begin{aligned}
\Delta p_{a}= & \left\{\left(n_{x}^{a}\right)^{2}+\left[1-\left(n_{x}^{a}\right)^{2}\right] \cos \lambda_{a}\right\} \\
& \times \prod_{b \in \mathcal{N}_{a}}\left\{\left(n_{z}^{b}\right)^{2}+\left[1-\left(n_{z}^{b}\right)^{2}\right] \cos \lambda_{b}\right\},
\end{aligned}
$$

which was supposed to give the difference in probability of obtaining +1 and -1 outcomes when measuring a correlator $K_{a}=X_{a} \bigotimes_{b \in \mathcal{N}_{a}} Z_{b}$ on a perturbed graph state. This formula is, in general, incorrect: It only represents one of a series of terms.

In this Erratum, we correct Eq. (6) in the original paper and derive some conditions under which the original expression still holds: In many of the cases considered in the original paper, the extra terms vanish. The high-level conclusions, as outlined in the abstract of the original article, are not affected.

\section{General correction of Eq. (6) in the original paper}

We consider a graph state perturbed by coherent noise, which we interpret as a stray field: $|\widetilde{G}\rangle:=U_{\text {tot }}|G\rangle$, where $U_{\text {tot }}=\bigotimes_{a \in V} U_{a}=\bigotimes_{a \in V} e^{-i \lambda_{a} \hat{n}_{a} S_{a} / 2}$. The quantity we want to evaluate is as follows:

$$
\begin{aligned}
\Delta p_{a} & =\left\langle\widetilde{G}\left|K_{a}\right| \widetilde{G}\right\rangle \\
& =\operatorname{Tr}\left[|G\rangle\langle G| U_{\mathrm{tot}}^{\dagger} K_{a} U_{\mathrm{tot}}\right] \\
& =\operatorname{Tr}\left[|G\rangle\langle G| K_{a}^{\dagger} U_{\mathrm{tot}}^{\dagger} K_{a} U_{\mathrm{tot}}\right] .
\end{aligned}
$$

First, we expand $|G\rangle\langle G|$ as a sum of stabilizer operators using the identity derived in Proposition 3 of Ref. [1],

$$
|G\rangle\langle G|=\frac{1}{2^{N}} \sum_{\xi \in \mathcal{S}} \xi=\frac{1}{2^{N}} \sum_{\substack{\left(j_{1}, \ldots, j_{N}\right) \\ \in\{0,1\}^{N}}} K_{1}^{j_{1}} \cdots K_{N}^{j_{N}},
$$

where $K_{a}^{0}=\mathbb{1}, K_{a}^{1}=K_{a}$, and the stabilizer group $\mathcal{S}$ is the set of elements $\xi$ of the multiqubit Pauli group $\mathcal{P}_{V}$ that stabilizes $|G\rangle, \xi|G\rangle=|G\rangle$. That is, $|G\rangle\langle G|$ is equal to the sum over all stabilizers of $|G\rangle$, and each stabilizer can be factorized uniquely as a product of correlators of $|G\rangle$.

Second, we expand $K_{a}^{\dagger} U_{\text {tot }}^{\dagger} K_{a} U_{\text {tot }}$ as a linear combination of Pauli operators. The single-qubit unitaries $U_{c}$ acting on qubits $c$ outside of $\mathcal{N}_{a}^{\prime}:=\mathcal{N}_{a} \cup\{a\}$ commute with $K_{a}$, and these operators cancel each other out. Consequently, $K_{a}^{\dagger} U_{\text {tot }}^{\dagger} K_{a} U_{\text {tot }}$ has support on (i.e., acts nontrivially only on) the qubits in the

$$
\begin{aligned}
& \text { set } \mathcal{N}_{a}^{\prime}, \\
& \qquad \begin{aligned}
K_{a}^{\dagger} U_{\text {tot }}^{\dagger} K_{a} U_{\text {tot }}= & K_{a}^{\dagger}\left(\bigotimes_{c \in \mathcal{N}_{a}^{\prime}} U_{c}^{\dagger}\right) K_{a}\left(\bigotimes_{d \in \mathcal{N}_{a}^{\prime}} U_{d}\right) \\
= & \left(\alpha_{\mathbb{1}}^{a} \mathbb{1}_{a}+i \alpha_{Y}^{a} Y_{a}+i \alpha_{Z}^{a} Z_{a}\right) \\
& \times \bigotimes_{b \in \mathcal{N}_{a}}\left(\tilde{\alpha}_{\mathbb{1}}^{b} \mathbb{1}_{b}+i \tilde{\alpha}_{X}^{b} X_{b}+i \tilde{\alpha}_{Y}^{b} Y_{b}\right) \bigotimes_{c \in V \backslash \mathcal{N}_{a}^{\prime}} \mathbb{1}_{c}
\end{aligned}
\end{aligned}
$$

The coefficients $\alpha_{1}^{a}, \alpha_{Y}^{a}, \alpha_{Z}^{a}, \tilde{\alpha}_{1}^{b}, \tilde{\alpha}_{X}^{b}$, and $\tilde{\alpha}_{Y}^{b}$ depend on the parameters of the field at vertex $a$ and at its neighbors $b \in \mathcal{N}_{a}$, and they can be computed expanding the expression in Eq. (3) as performed in Appendix A of the original article,

$$
\begin{aligned}
\alpha_{1}^{a} & =\left(n_{x}^{a}\right)^{2}+\left[1-\left(n_{x}^{a}\right)^{2}\right] \cos \left(\lambda_{a}\right), \\
\alpha_{Y}^{a} & =-n_{y}^{a} \sin \left(\lambda_{a}\right)-n_{x}^{a} n_{z}^{a}\left[1-\cos \left(\lambda_{a}\right)\right], \\
\alpha_{Z}^{a} & =-n_{z}^{a} \sin \left(\lambda_{a}\right)+n_{x}^{a} n_{y}^{a}\left[1-\cos \left(\lambda_{a}\right)\right], \\
\tilde{\alpha}_{1}^{b} & =\left(n_{z}^{b}\right)^{2}+\left[1-\left(n_{z}^{b}\right)^{2}\right] \cos \left(\lambda_{b}\right), \\
\tilde{\alpha}_{X}^{b} & =-n_{x}^{b} \sin \left(\lambda_{b}\right)-n_{y}^{b} n_{z}^{b}\left[1-\cos \left(\lambda_{b}\right)\right], \\
\tilde{\alpha}_{Y}^{b} & =-n_{y}^{b} \sin \left(\lambda_{b}\right)+n_{x}^{b} n_{z}^{b}\left[1-\cos \left(\lambda_{b}\right)\right] .
\end{aligned}
$$

Equation (4) can be compactly written as

$$
K_{a}^{\dagger} U_{\text {tot }}^{\dagger} K_{a} U_{\text {tot }}=\sum_{\xi \in \mathcal{P}_{\mathcal{N}_{a}^{\prime}}} c_{\xi}^{a} \xi,
$$

where the set $\mathcal{P}_{\mathcal{N}_{a}^{\prime}}=\left\{\xi \in\{\mathbb{1}, X, Y, Z\}^{\otimes N} \mid \xi_{c}=\mathbb{1}_{c} \forall c \notin \mathcal{N}_{a}^{\prime}\right\}$ consists of multiqubit Pauli operators that act as the identity outside of the set $\mathcal{N}_{a}^{\prime}$ and $c_{\xi}^{a} \in \mathbb{C}$ denotes a product of $\alpha_{1}^{a}, i \alpha_{Y}^{a}, i \alpha_{Z}^{a}, \tilde{\alpha}_{1}^{b}, i \tilde{\alpha}_{X}^{b}$, and $i \tilde{\alpha}_{Y}^{b}$.

Using the expressions now given for $|G\rangle\langle G|$ and $K_{a}^{\dagger} U_{\text {tot }}^{\dagger} K_{a} U_{\text {tot }}$ and the linearity of the trace, Eq. (1) becomes

$$
\Delta p_{a}=\sum_{\substack{\left(j_{1}, \ldots, j_{N}\right) \\ \in\{0,1\}^{N}}} \sum_{\xi \in \mathcal{P}_{\mathcal{N}_{a}^{\prime}}} \frac{c_{\xi}^{a}}{2^{N}} \operatorname{Tr}\left[K_{1}^{j_{1}} \cdots K_{N}^{j_{N}} \xi\right] .
$$

We then use $\operatorname{Tr}[\zeta]=2^{N} \delta_{\zeta, \mathbb{1}}$ for $\zeta \in\{\mathbb{1}, X, Y, Z\}^{\otimes N}$. The methods given in Sec. 2 of Ref. [1] can be used to show that, if $K_{1}^{j_{1}} \cdots K_{N}^{j_{N}} \xi=\mu \mathbb{1}^{\otimes N}$, then the phase is $\mu= \pm 1$ and, therefore, $K_{1}^{j_{1}} \cdots K_{N}^{j_{N}}=\mu_{\xi} \xi$, with $\mu_{\xi} \in\{-1,+1\}$. Hence,

$$
\Delta p_{a}=\sum_{\xi \in \mathcal{S} \cap \pm \mathcal{P}_{\mathcal{N}_{a}^{\prime}}} \mu_{\xi} c_{\xi}^{a} \text {. }
$$


That is, the sum in Eq. (8) runs over multiqubit Pauli operators that satisfy two requirements: First, they are stabilizer operators; second, these operators act nontrivially only on the qubits in the set $\mathcal{N}_{a}^{\prime}$. There are always, at least, two operators that satisfy both requirements: $\mathbb{1}^{\otimes N}$ and $K_{a}$. If $\mathbb{1}^{\otimes N}$ and $K_{a}$ are the only stabilizers with support contained in $\mathcal{N}_{a}^{\prime}$, then we have $\Delta p_{a}=c_{\mathbb{1}}^{a}+c_{K_{a}}^{a}=c_{\mathbb{1}}^{a}$ since Eq. (4) implies $c_{K_{a}}^{a}=0$.

In conclusion, Eq. (6) in the original paper is correct exactly when Eq. (8) consists of only one term,

$$
\begin{aligned}
\Delta p_{a}= & c_{\mathbb{1}}^{a}=\alpha_{\mathbb{1}}^{a} \prod_{b \in \mathcal{N}_{a}} \tilde{\alpha}_{\mathbb{1}}^{b}=\left\{\left(n_{x}^{a}\right)^{2}+\left[1-\left(n_{x}^{a}\right)^{2}\right] \cos \lambda_{a}\right\} \\
& \times \prod_{b \in \mathcal{N}_{a}}\left\{\left(n_{z}^{b}\right)^{2}+\left[1-\left(n_{z}^{b}\right)^{2}\right] \cos \lambda_{b}\right\}
\end{aligned}
$$

However, Eq. (9) does not always hold as other nonzero coefficients can be present in Eq. (8). A counterexample is given by the two-qubit graph state $|G\rangle=C Z_{1,2}|+\rangle_{1}|+\rangle_{2}$, which has stabilizers $\mathbb{1} \otimes \mathbb{1}, X \otimes Z, Z \otimes X$, and $Y \otimes Y$ so that we have

$$
\begin{aligned}
& \Delta p_{1}=\left\langle\widetilde{G}\left|X_{1} \otimes Z_{2}\right| \widetilde{G}\right\rangle=c_{1 \otimes \mathbb{1}}^{1}+c_{X \otimes Z}^{1}+c_{Z \otimes X}^{1}+c_{Y \otimes Y}^{1}, \\
& \Delta p_{2}=\left\langle\widetilde{G}\left|Z_{1} \otimes X_{2}\right| \widetilde{G}\right\rangle=c_{1 \otimes \mathbb{1}}^{2}+c_{X \otimes Z}^{2}+c_{Z \otimes X}^{2}+c_{Y \otimes Y}^{2} .
\end{aligned}
$$

From Eq. (4), it follows that $c_{X \otimes Z}^{1}=c_{Z \otimes X}^{2}=0$, but the other coefficients are, in general, different from zero.

\section{Conditions for correctness of Eq. (6) in the original paper}

We now show, following two lines of reasoning, that in many of the cases analyzed in the original article, Eq. (6) in the original paper still holds. First, we give conditions under which $\mathbb{1}$ and $K_{a}$ are the only stabilizers with support contained in $\mathcal{N}_{a}^{\prime}$. Second, we show that, even if other stabilizers $\xi$ have support contained in $\mathcal{N}_{a}^{\prime}$, their coefficients $c_{\xi}^{a}$ can still be zero because of the specific form of Eq. (4).

First, a stabilizer $\xi$ has support contained in $\mathcal{N}_{a}^{\prime}$ when its factorization $\xi=K_{1}^{j_{1}} \cdots K_{N}^{j_{N}}$ consists of a product of correlators $K_{c}$ each centered at a qubit $c \in \mathcal{N}_{a}^{\prime}$. This is the case because a correlator $K_{c}$ has the form $K_{c}=X_{c} \bigotimes_{d \in \mathcal{N}_{c}} Z_{d}$ and all other correlators either act as $\mathbb{1}_{c}$ or as $Z_{c}$ on the qubit at the vertex $c$; thus, they cannot cancel out the $X_{c}$ operator appearing in $K_{c}$. Therefore, the sum in Eq. (8) contains at most $2^{d_{a}}$ terms, where $d_{a}=\left|\mathcal{N}_{a}^{\prime}\right|$ is the number of vertices in $\mathcal{N}_{a}^{\prime}$ : Only the stabilizers that are products of correlators $K_{c}$ with $c \in \mathcal{N}_{a}^{\prime}$ can contribute. For a given subset $M \subseteq \mathcal{N}_{a}^{\prime}$, the operator $\prod_{a \in M} K_{a}$ has support contained in $\mathcal{N}_{a}^{\prime}$ if and only if all the $Z$ operators acting on qubits outside of $\mathcal{N}_{a}^{\prime}$ cancel each other out. This happens exactly when each vertex $c \notin \mathcal{N}_{a}^{\prime}$ is adjacent to an even number of vertices belonging to $M$. Using this fact, in certain cases we can prove that $\mathbb{1}$ and $K_{a}$ are the only stabilizers with support contained in $\mathcal{N}_{a}^{\prime}$. In particular, this holds (for all $a \in V$ ) for square lattices with sides of length $m_{1} \geqslant 4$ and $m_{2} \geqslant 2$ for planar geometry and for square lattices with $m_{1} \geqslant 5$ and $m_{2} \geqslant 3$ for toroidal geometry. Hence, using $c_{K_{a}}^{a}=0$, in all these cases, we have $\Delta p_{a}=c_{\mathbb{1}}^{a}$, and thus Eq. (6) in the original paper holds.

Second, we use Eq. (4) to show that, when the stray fields are parallel to one of the Cartesian axes, some simplifications take place, often resulting in Eq. (6) in the original paper being still valid. For instance, for $Z$ fields, we have $\Delta p_{a}=\cos \lambda_{a}$ for all graph states. Thus, as stated in the original article, $Z$ fields are always reconstructible. For $X$ and $Y$ fields we have

X field: $\quad K_{a}^{\dagger} U_{\text {tot }}^{\dagger} K_{a} U_{t}$

$$
=\bigotimes_{b \in \mathcal{N}_{a}}\left[\cos \left(\lambda_{b}\right) \mathbb{1}_{b}-i \sin \left(\lambda_{b}\right) X_{b}\right] \bigotimes_{c \in V \backslash \mathcal{N}_{a}} \mathbb{1}_{c},
$$

Y field: $K_{a}^{\dagger} U_{\text {tot }}^{\dagger} K_{a} U_{\text {tot }}$

$$
\begin{aligned}
= & {\left[\cos \left(\lambda_{a}\right) \mathbb{1}_{a}-i \sin \left(\lambda_{a}\right) Y_{a}\right] } \\
& \times \bigotimes_{b \in \mathcal{N}_{a}}\left[\cos \left(\lambda_{b}\right) \mathbb{1}_{b}-i \sin \left(\lambda_{b}\right) Y_{b}\right] \bigotimes_{c \in V \backslash \mathcal{N}_{a}^{\prime}} \mathbb{1}_{c} .
\end{aligned}
$$

As a consequence, many coefficients $c_{\xi}^{a}$ vanish

X field: $\quad c_{\xi}^{a}=0$,

if $\xi_{a} \notin\left\{\mathbb{1}_{a}\right\}$, or if $\xi_{b} \notin\left\{\mathbb{1}_{b}, X_{b}\right\}$ for some $b \in \mathcal{N}_{a}$,

Y field: $\quad c_{\xi}^{a}=0$,

$$
\begin{aligned}
& \text { if } \xi_{a} \notin\left\{\mathbb{1}_{a}, Y_{a}\right\}, \text { or } \\
& \text { if } \xi_{b} \notin\left\{\mathbb{1}_{b}, Y_{b}\right\} \text { for some } b \in \mathcal{N}_{a} .
\end{aligned}
$$

These two sets of simplifications are crucial for linear chains (i.e., one-dimensional cluster states). First, consider closed linear chains with $m \geqslant 5$ qubits. In this case, we have $\Delta p_{a}=$ $c_{\mathbb{1}}^{a}$ for all vertices. In fact, the set of stabilizer operators having support on $\mathcal{N}_{a}^{\prime}$ consists only of $\mathbb{1}$ and $K_{a}$ since the correlators $K_{a-1}$ and $K_{a+1}$ act as $Z$ on the vertices in positions $a-2$ and $a+2$, and, for chains of length greater than 4, these $Z$ operators cannot cancel each other out. Next, consider open-ended linear chains with $m \geqslant 4$ qubits (numbered in the obvious way): For each vertex $a$, except for $a=2$ and $a=m-1$, only $\mathbb{1}$ and $K_{a}$ have support on $\mathcal{N}_{a}^{\prime}$. We then have

$$
\begin{array}{ll}
\Delta p_{a}=c_{\mathbb{1}}^{a}, & \text { if } a \notin\{2, m-1\}, \\
\Delta p_{a}=c_{\mathbb{1}}^{a}+c_{X_{1} \otimes Z_{2}}^{a}, & \text { if } a=2, \\
\Delta p_{a}=c_{\mathbb{1}}^{a}+c_{Z_{m-1} \otimes X_{m}}^{a}, & \text { if } a=m-1 .
\end{array}
$$

Hence, Eq. (6) in the original paper does not generically hold for open-ended linear chains. But specializing to the $X$ - and $Y$-field cases, we have $\Delta p_{a}=c_{\mathbb{1}}^{a}$ for all the vertices $a \in V$, hence, for all these cases, the analyses in the original article are correct.

\section{Correction of tables and plots}

Several cases considered in the original article have to be analyzed on an individual basis. These computations are lengthy but straightforward. Here, we show how the plots and the tables in the original article have to be modified when using the correct formula Eq. (8) instead of Eq. (6) in the original paper. We present here corrected versions of Tables I and III and Figs. 1-3. The main differences with the original tables and plots are described in the captions.

\section{ACKNOWLEDGMENT}

We thank G. Glesener and K. Ried for helpful discussions and feedback. 


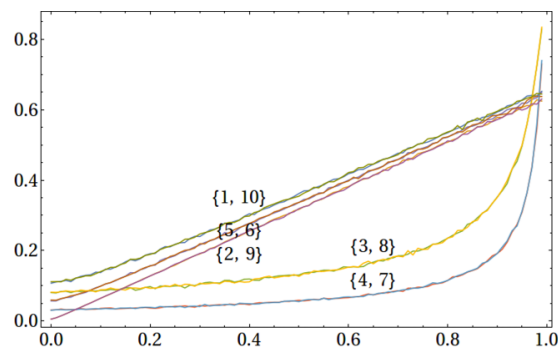

$X$-field, $q$-dependence

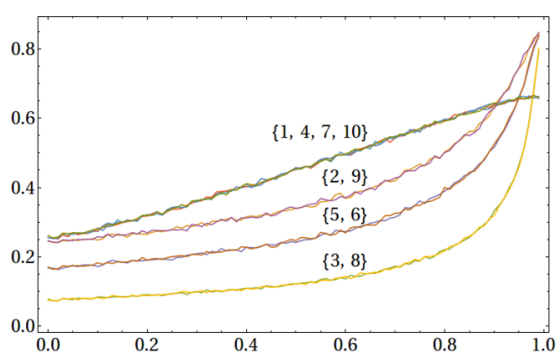

$Y$-field, $q$-dependence

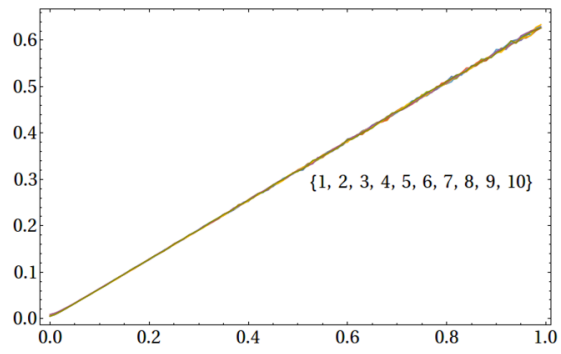

$Z$-field, $q$-dependence

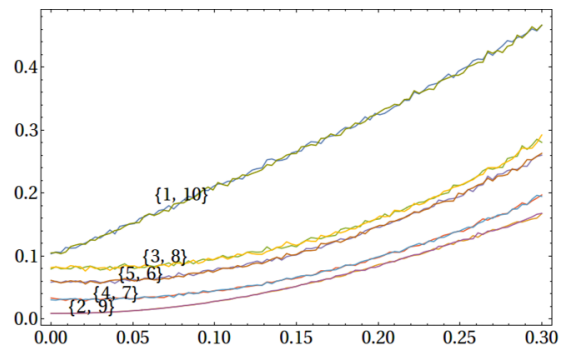

$X$-field, $\epsilon$-dependence

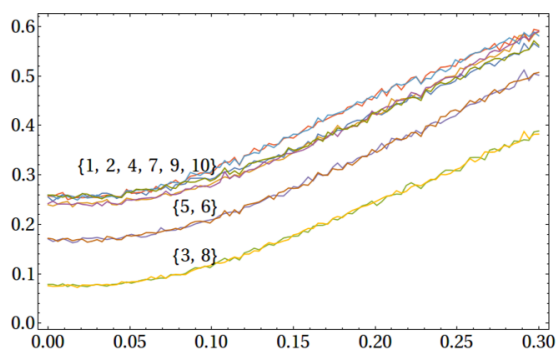

$Y$-field, $\epsilon$-dependence

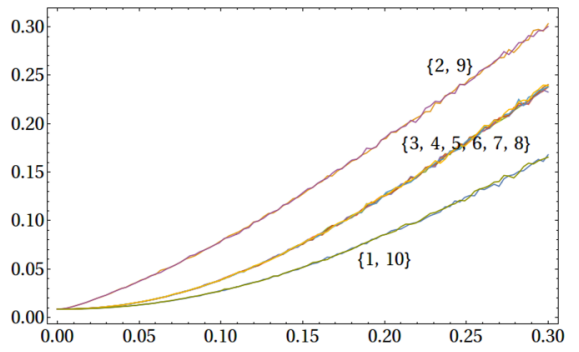

$Z$-field, $\epsilon$-dependence

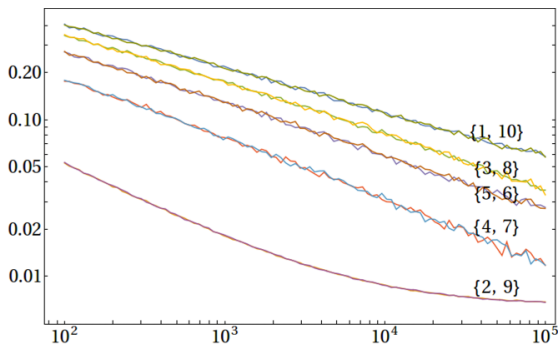

$X$-field, $M$-dependence

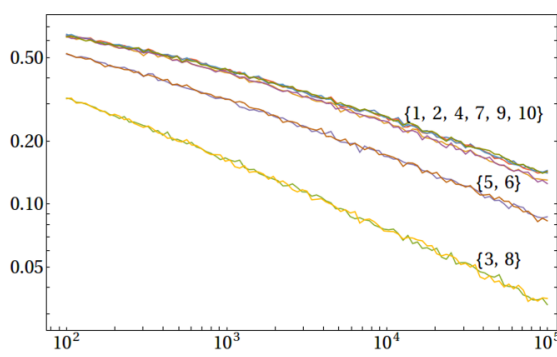

$Y$-field, $M$-dependence

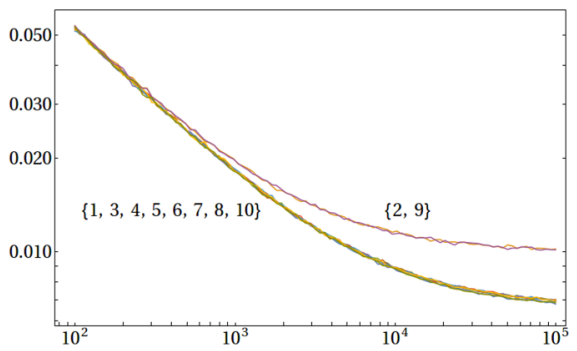

$Z$-field, $M$-dependence

FIG. 1. The plots are all qualitatively similar to the plots in the original article except for three cases. In panel ( $X$ field, $\epsilon$ dependence), qubits 1 and 10 have a larger reconstruction error than what was previously displayed. In panel ( $Z$ field, $\epsilon$ dependence), qubits 2 and 9 have a larger reconstruction error than what was previously displayed (the curves for qubits 2 and 9 before were essentially overlapping with the curves for qubits 3-8). In panel ( $Z$ field, $\epsilon$ dependence), qubits 2 and 9 have a larger reconstruction error than what was previously displayed (the curves for all qubits were essentially overlapping).

TABLE I. We consider linear chains of length $m \in \mathbb{N}$. In each entry of the table, we give the set of values of $m$ such that $\Delta p_{a}=c_{1}^{a}$ holds $\forall a$ and, hence, Eq. (6) in the original paper is correct. $Z$ fields can always be reconstructed.

\begin{tabular}{lcc}
\hline \hline & $\begin{array}{c}\text { Open-end } \\
\text { linear chain }\end{array}$ & $\begin{array}{c}\text { Closed } \\
\text { linear chain }\end{array}$ \\
\hline General field & No $m$ & $m \geqslant 5$ \\
$X$ field & $m \neq 1,3$ & $m \neq 1,4$ \\
$Y$ field & $m \neq 2$ & $m \neq 2,3$ \\
\hline \hline
\end{tabular}

TABLE III. We consider fully connected graphs and star graphs with $N$ vertices (both representing GHZ states up to local unitaries). In each entry of the table, we give the set of values of $N \in \mathbb{N}$ such that $\Delta p_{a}=c_{\mathbb{1}}^{a}$ holds $\forall a$.

\begin{tabular}{lcc}
\hline \hline field & $N$ & No $N$ \\
$Y$ field & $N \geqslant 2$ & No $N$ \\
Eoneral field $N$ & Even $N$ \\
\hline
\end{tabular}




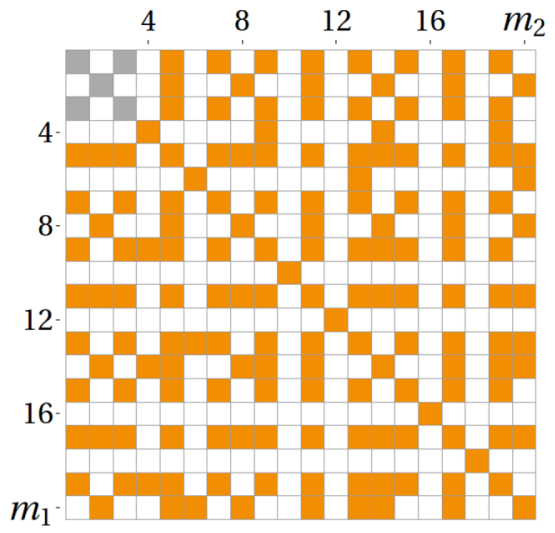

$X$-field, planar

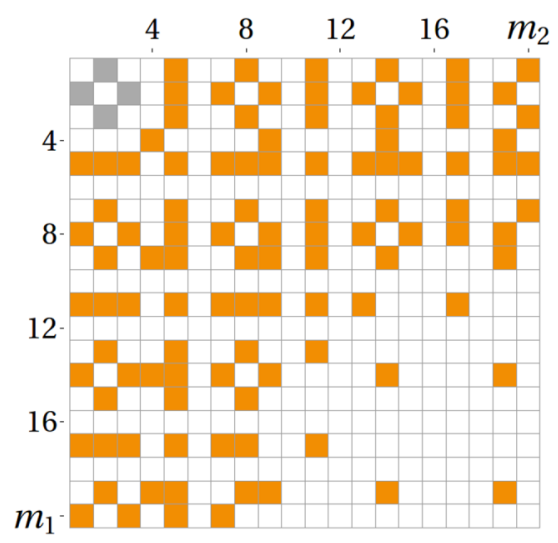

$Y$-field, planar

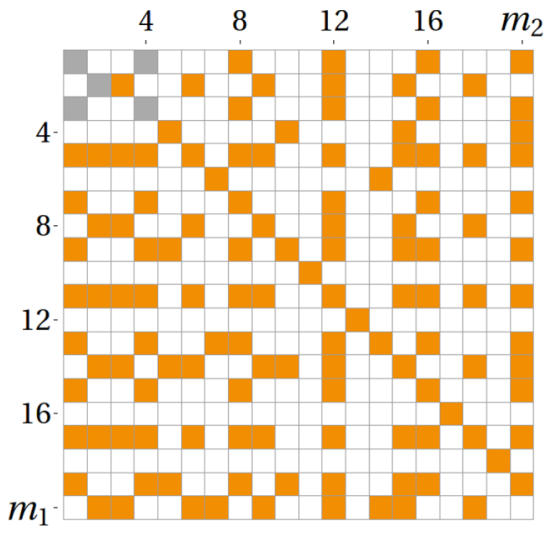

$X$-field, cylindrical

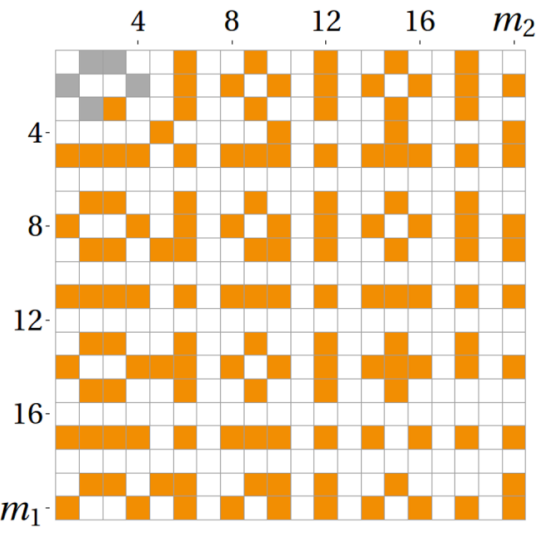

$Y$-field, cylindrical

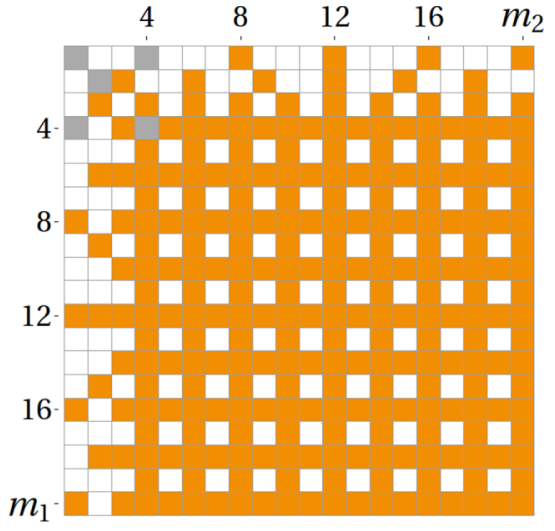

$X$-field, toroidal

FIG. 2. The gray cells correspond to the cases where Eq. (6) in the original paper is not valid, and, thus, the reconstruction methods are not applicable in these cases. All other cases remain unaltered.

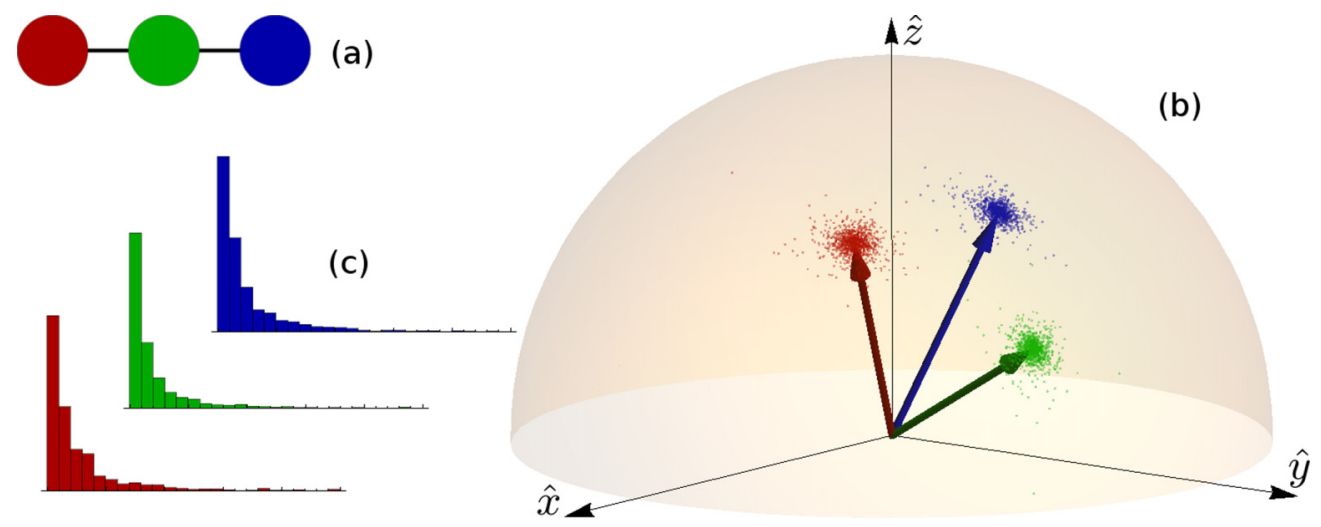

FIG. 3. These are the results of the numerical analyses for field reconstruction including the correction of Eq. (6) in the original paper. It seems that an anisotropy in the dispersion of the data points for the reconstructed fields has been removed.

[1] M. Hein, W. Dür, J. Eisert, R. Raussendorf, M. Van den Nest, and H. J. Briegel, Entanglement in graph states and its applications,
IOS Press Proceedings of the International School of Physics "Enrico Fermi" 162, 115 (2006). 